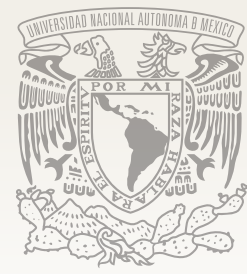

\title{
Revista
}

Pueblos y fronteras

\section{MÁS ALLÁ DEL CENTRO Y LA PERIFERIA: \\ LA FRONTERA SUR DE MÉXICO A DEBATE DESDE LA GLOBALIZACIÓN}

Hanna Laako

laako.hanna@gmail.com

Becaria del Programa de Becas Posdoctorales en la UNAM, Instituto de Investigaciones Antropológicas, PROIMMSE-UNAM

\section{RESUMEN}

El artículo examina analíticamente el concepto y la realidad vivida de la frontera sur de México en el contexto de los debates contemporáneos sobre las fronteras con el objetivo de indagar qué tan periférica sigue siendo actualmente. Indagando la llamada tesis de la globalización sin fronteras, se argumenta que ésta logró ampliar el entendimiento de la frontera más allá del estado-centrismo y el eurocentrismo, permitiendo también repensar la frontera sur de México más allá de los centros y las periferias geográfico-nacionales.

Palabras clave: globalización, relaciones internacionales, frontera, frontera sur de México, colonización, migración, regiones.

\section{BEYOND THE CENTER AND THE PERIPHERY: MEXICO'S SOUTHERN BORDER UNDER DEBATE FROM A GLOBALIZATION PERSPECTIVE}

\section{ABSTRACT}

The article analytically revisits the concept and reality lived in the southern border/ frontier of Mexico in the context of contemporary Borderlands debates, inquiring how peripheral the region continues to be today. Analyzing the so called borderless globalization thesis, it is argued that the mentioned thesis managed to extend the understanding of the border beyond the previous state-centrism and euro-centrism. This could permit to rethink what we understand as the Mexican southern border today beyond the national-geographic centers and peripheries.

Key words: globalization, international relations, border, borderlands, southern Mexican frontier, colonization, migration. 


\section{INTRODUCCIÓN}

Varios autores coinciden en que la década de 1980 puso a la vista la necesidad de mover la atención que estaba puesta hasta ese entonces en forma dominante en la frontera norte y empezar a dirigir la mirada hacia el límite territorial del sur de México. Derivado de ello, esta frontera geográfica empezó a convertirse en objeto de estudio, y como resultado ello se establecieron varias instancias académicas en la región (Fábregas 2011, Kauffer 2002).²

En los estudios iniciales (Fábregas et al. 1985, Chenaut 1989, De Vos 1993) la frontera sur de México aparecía como un área periférica, aislada y olvidada pero no carente de procesos históricos importantes. Sin embargo, en la década de los noventa, fenómenos como el de las movilizaciones indígenas y el de una creciente migración de refugiados e indocumentados atrajeron la atención nacional e internacional, por lo que la cantidad de estudios vinculados a temas relacionados con esta zona fronteriza se ha incrementado exponencialmente. A la luz de lo señalado, es factible preguntarse sobre qué tan periférica sigue siendo esta frontera, sobre todo teniendo en cuenta la predominancia de la visión mexicana sobre la centroamericana y respecto del rol del estado de Chiapas en comparación con las

\footnotetext{
${ }^{1}$ Este artículo se basa en una investigación posdoctoral llevada a cabo durante 2013 en el Colegio de la Frontera Sur (ECOSUR). Partes diferentes del artículo han sido presentadas como ponencias en tres conferencias recientes: 1) en el Segundo Coloquio Regionaly el Primer Congreso Internacional "Género y Fronteras: Limites, frentes y espacios», celebrados en San Cristóbal de Las Casas, Chiapas, del 25 al 27 de septiembre de 2013; 2) en el Encuentro del Departamento Sociedad, Cultura y Salud de El Colegio de la Frontera Sur, celebrado en San Cristóbal de Las Casas, Chiapas, del 10 al 12 de diciembre de 2013, y 3) en el taller temático «Cuencas Transfronterizas: espacios de expresión de lo político", celebrado en la ciudad de México, cIESAs, del $15 \mathrm{al}$ 17 de enero de 2014. Agradezco a las doctoras Guadalupe Álvarez y Celia Ruiz de la Oña y al doctor Manuel Martínez Espinoza por sus sugerencias tan constructivas en la elaboración esta versión del artículo, así como a los comentaristas de las ponencias y los dictaminadores del artículo para su publicación.

${ }^{2}$ Según Fábregas et al. (1985), la región sureste de México se refiere al territorio que cubren los estados de Campeche, Chiapas, Quintana Roo, Tabasco y Yucatán, de los cuales solo Yucatán no es directamente fronterizo con otro país, sino representa un estado fronterizo del mar.
}

otras entidades limítrofes. ¿Qué significados se le han dado al concepto de frontera y específicamente en el caso de la del sur de México durante las últimas décadas? ¿Cómo puede contribuir al análisis de ese concepto para entender la región?

Aunque se trata de una línea divisoria concreta entre México, Guatemala y Belice, y constituye una realidad vivida, representa también, al mismo tiempo, un concepto producido desde las instancias académicas, a partir de la década de los ochenta, y es además una región con características particulares. Los debates sobre su significado se han expresado en forma simultánea a las discusiones que tuvieron lugar en los años noventa, en las ciencias sociales, respecto de las fronteras de la globalización. Una característica primordial de este debate era la tesis de que la globalización había hecho desaparecer la relevancia de las fronteras, y se argumentaba que a partir de esta, la política mundial tenía ya una condición sin fronteras, que se evidenciaba en el contexto de los Estados-nación modernos, las empresas multinacionales y los movimientos sociales globales. Además, su papel decreciente se comprobaba en los flujos de información y de capital global, así como en la emergencia de la Unión Europea después de la «caída de los muros», con el consecuente libre tránsito de las personas. Desde este punto de vista, se consideraba que en su forma tradicional de fronteras físicas divisorias entre los Estados-nación, habían perdido su relevancia dado que muchos actores políticos contemporáneos eran de carácter no estatal y su accionar político traspasaba los límites tradicionales. Entonces, se planteaba la tesis optimista de un mundo sin fronteras, que se vinculaba fundamentalmente en una definición clásica y algo estática (véanse, por ejemplo, Hirst y Thompson 1999, Hardt y Negri 2000, Keck y Sikkink 1998, Newman 2006a, 2006b).

En las últimas décadas el multicitado concepto ha emergido de nuevo en las discusiones teóricas, incluso con su propio campo de investigación denominado Border Studies o Borderlands Studies (véanse, por ejemplo, Paasi 2005, Newman 2006a, 2006b, Sohi 2011). ${ }^{3}$ De acuerdo

${ }^{3}$ Según Newman (2006a, 2006b) y Paasi (2005), la emergencia contemporánea de estudios sobre fronteras o regiones fronterizas se puede observar en varios ejes: en la 
con Newman (2006a, 2006b), la discusión actual cuestiona el planteamiento de la globalización sin fronteras al explorar el tipo de demarcaciones o de procesos políticos contemporáneos transfronterizos que se observan en la actualidad. Según dicho autor, la crítica sirvió para renovar y despertar el interés por explorar las fronteras contemporáneas del mundo, en vez de concebir simplemente su desaparición.

En este artículo extiendo el razonamiento de Newman y argumento que el debate sobre la globalización y las fronteras ha contribuido a un mayor entendimiento de estas en varios sentidos, aun sin aceptar el planteamiento de su desaparición. En primer lugar ha permitido ampliar las definiciones desde su concepto clásico de líneas entre Estados, el cual, sin perder su relevancia, ha dejado de ser objeto de estudios estáticos y poco explicativos. En segundo lugar, admitió incluir a otros actores y perspectivas relacionados: los actores no estatales y su rol en la formación de los fenómenos fronterizos. De este modo se puede observar que desde el cuestionamiento de la tesis de la globalización y el renovado entusiasmo intelectual sobre las fronteras se ha permitido la deconstrucción del concepto anterior para repensar sus significados más allá de los centros y periferias nacionales y geográficos.

Analizo, en síntesis, los debates sobre la frontera sur de México y las distintas acepciones del concepto en las ciencias sociales. Examino con una mirada crítica la mencionada tesis de la globalización y sus contribuciones a la exploración de las fronteras actuales. Después, la percepción de la frontera en las ciencias sociales, para mostrar la ampliación de sus definiciones.

\section{FRONTERAS VIVIDAS, FRONTERAS CONSTRUIDAS: EL CASO DE LA FRONTERA SUR DE MÉXICO}

Como se mencionó anteriormente, el concepto y la realidad de la frontera sur de México se

cantidad de estudios y publicaciones sobre las fronteras, la cantidad de centros de investigación establecidos sobre la exploración de las fronteras, el interés de los financiadores y editoriales por publicar sobre las fronteras, y también en el interés académico de cruzar sus propias fronteras para colaborar en una forma multidisciplinaria sobre las fronteras contemporáneas. replanteó en la década de los ochenta debido a varios procesos sociopolíticos acontecidos en esta región que, al llamar la atención académica y política, contrastaron con la visión dominante orientada principalmente al estudio de la línea que divide a México y a los Estados Unidos. Sobre todo, destaca el trabajo de Fábregas et al. (1985) en el cual se explora profundamente la formación histórica de la región sureste en colindancia. Explica De Vos (2002) que este interés empezó a finales de la década de 1980 con la introducción de este concepto/realidad en los medios políticos y académicos, en los cuales se inquiría sobre sus implicaciones históricas. Según ese autor, antes se habían hecho referencias al sureste utilizando esta categoría para nombrar al conjunto geopolítico que reunía los cinco estados más meridionales de México: Yucatán, Campeche, Quintana Roo, Tabasco y Chiapas. Pero a partir de los ochenta se evidenció un proceso de descubrimiento de la situación fronteriza de esa región y su importancia para las ciencias sociales. ${ }^{4}$

Fábregas (1997a) explica el concepto de frontera en el caso del sur de México con base en cuatro ejes. Primero, como un lugar de expansión y un área geográfica relativamente despoblada en la que un bajo radio en la relación hombre-tierra, más una abundancia de recursos naturales, le otorgan al Estado nacional la oportunidad de fortalecerse y resolver diferentes presiones sociales. En este aspecto se puede decir que la frontera se vincula con la colonización y con el estadocentrismo en sus diferentes sentidos. Fábregas argumenta que el trópico húmedo del sur mexicano ha sido una frontera en el sentido de ocupación para el propio Estado nacional mexicano, además de lugar de establecimiento de los límites políticos de la nación. En segundo lugar, se expresa como un espacio de economía regional, surgido desde los estudios de la econo-

\footnotetext{
${ }^{4}$ Durante esta década se establecieron varias instancias de investigación dedicadas a la exploración de la región sureste y la frontera sur, entre ellas, El Colegio de la Frontera Sur (ECOSUR) y el Centro de Investigaciones y Estudios Superiores en Antropología Unidad Sureste (CIESAS-Sureste), ambos con interés especial en problemáticas fronterizas. Véase Kauffer (2002) y la página de web del CIESAS-Sureste (consultada en mayo de 2013): http://sureste.ciesas. edu.mx/
} 
mía de frontera en el Río Bravo, entre el sur de Estados Unidos y el norte de México. Aquí, la frontera se confirma en la movilidad comercial o de trabajo, que también marca la historia de los ciclos agrícolas en el Soconusco en Chiapas. En tercer lugar, la frontera se entiende también como un espacio de convergencias, sobre todo sociales y culturales, a lo largo de una historia particular. Según Fábregas, se puede hablar de espacios plurinacionales cuyo contenido no está en la economía, sino en redes de relaciones sociales y en afinidades culturales. Básicamente, la frontera es un territorio común. Y por último, Fábregas analiza el sentido de la frontera como el límite tradicional: una línea que se traza para marcar un orden, "este lado» y «el otro lado», «antes» y «después». En su forma más tradicional, la frontera-límite es la expresión de una forma de ejercer el poder y de concebir un «territorio político». Sin embargo, el límite es también una relación, muchas veces dialéctica. Así, la concepción de la frontera en México también forma parte de una ideología que enfatiza el nacionalismo, y a este como la expresión del proyecto propio de sociedad y de cultura.

También se puede observar en los estudios iniciales el giro que representó cambiar la atención desde lo hegemónico -la frontera norte- hacia la periférica, marginal, aislada, poco desarrollada, politica e históricamente complicada frontera del sur de México. Para los investigadores y las instancias académicas en la frontera del sur se marca un territorio en el que coexisten diferentes ámbitos regionales que han sido resultado de procesos sociales iniciados hace 400 años. Por ejemplo, según Saldívar y Arreola (1997: 239), esta frontera es como una «unidad territorial, ambiental e incluso cultural en donde la frontera sur aparece como una de las regiones del país en donde, a pesar de la gran atención institucional, la mayoría de las decisiones importantes siguen tomándose desde fuera, y continúa existiendo una fuerte dependencia económica respecto al centro». De este modo, la frontera sur está caracterizada por una estructura de producción regional con un sistema de movimientos migratorios de importantes volúmenes de mano de obra barata que conviven con conceptos como geopolítica, vecindad, insurgencia, guerra, narcotráfico, po- breza, etnicidad, ecología y soberanía. Al mismo tiempo, esta frontera ha sido considerada como «olvidada», «marginada», "periférica», lo que muestra su definición como un lugar alejado del centro, tal vez místico o incluso vacío, lo cual marca un contraste con los estudios que enfocan la frontera sur como un espacio de varios fenómenos significativos y como un objeto de todo tipo de políticas y actores.

Sin embargo, según otros autores fue sobre todo la presencia de los refugiados guatemaltecos en el estado de Chiapas lo que influyó para atraer la atención nacional desde la frontera norte hacia el sur "periférico». El Estado mexicano comenzó a formular una política fronteriza para la región a partir de la década de 1980 con la llegada de los refugiados a su territorio y ante la agudización de la lucha armada en Guatemala. Pohlenz (1985) identifica el conflicto centroamericano como uno de los elementos más importantes que definen el perfil actual de la frontera sur de México. De igual manera, Fábregas (1997b) argumenta que en 1983 empieza un nuevo periodo de reflexión con la formación y las características de la frontera sur mexicana como un tema central, en donde los tejidos entre la migración/colonización de todo tipo, la cultura y la sociedad es lo que importa en el proceso de creación de nuevos territorios. En este punto, dos enfoques resaltan respecto del tema de la frontera sur: la colonización y la movilización indígena.

Según Leyva y Ascencio (1997), la colonización se puede vincular con la expansión de fronteras, la dominación, el control político-económico y las relaciones de centro y periferia en donde nuevas regiones entran en el proceso de globalización, en la formación del Estado y la nación. Según estos autores, los enfoques de colonización en el caso de Chiapas han tenido varios puntos de vista, desde el productivista-demográfico e histórico-estructural (en que la colonización se percibe como una migración interna rural-rural), hasta el socioagrario y sociocultural. Los enfoques de colonización muestran la amplitud del fenómeno en el que la migración solamente forma una parte de los procesos mencionados. 
La colonización y la realidad fronteriza también cobran importancia en el estudio de Chenaut (1989) sobre los migrantes y aventureros en la frontera sur, en este caso, en la frontera particular entre México y Belice, en el estado de Quintana Roo. En dicho estudio, realizado en el momento del «redescubrimiento de la frontera sur», Chenaut explora las comunidades del Río Hondo, no solamente como un límite sino como un centro fundamental de comunicación más allá del establecimiento de las fronteras nacionales. Como una frontera-frente, dicho autor destaca los migrantes y aventureros en la zona selvática del Río Hondo: mayas rebeldes, ingleses, chicleros, campesinos sin tierra, grupos étnicos diferentes, menonitas, beliceños, nativos, indios y negros. Su obra aborda los diferentes sentidos de la frontera «olvidada» pero sin el aislamiento, como una frontera en realidad poblada, colonizada y adoptada por los «perseguidos» y buscadores de un espacio autónomo para su existencia. Se pregunta después (1989: 92): «El límite social es el que imponen las ficticias divisiones políticas entre estados o países? Evidentemente no. En el caso que estamos analizando encontramos otro ejemplo que nos muestra que la frontera entre los países no es, a la vez, una frontera social. Desde este punto de vista no podemos pensar a este espacio más que en términos de una «región». ${ }^{5}$

De esta forma, para muchos investigadores del tema ha tenido más sentido hablar de la región sureste, en donde la frontera-límite marca solamente la historia más reciente del Estado-nación, si se toma en cuenta que esta ha sido poblada primordialmente por los pueblos mayas, pero se han vivido otras: las fronteras como procesos históricos que entrelazan la movilización de los pueblos, sus identidades y relaciones sociales (Castillo 2002: 19-49). Aquí se destaca el trabajo de De Vos (1993, 2002) que analiza la problemática desde el enfoque de las aspiraciones hegemónicas de diversos centros de poder que llegaron a demarcar sus respectivas zonas de influencia sobre el territorio de la región: la época prehis-

${ }^{5}$ El estudio de Chenaut cobra importancia especialmente por explorar la frontera de Belice y México en Quintana Roo, ya que el estado de Chiapas ha dominado los estudios de la frontera sur. pánica entre los pueblos mayas y la entrada de los aztecas, la conquista española, la consolidación de la colonia, la conquista inglesa en Belice, el proyecto mexicano de nación y el proyecto guatemalteco sobre el Soconusco en Chiapas.

De Vos (2002: 62) argumenta que el campesino maya deberá ocupar el lugar principal en los análisis de la frontera sur o región sureste, ya que constituye el único actor que perduró desde la época prehispánica hasta la actualidad. Por su parte, Kauffer (2002: 12) apunta que la realidad no indígena se halla pobremente representada. Considerando las fronteras étnicas del sur y el impacto de los movimientos indígenas en la región sureste, sería importante explorar las fronteras indígenas y no indígenas, entre el campo y la ciudad, debido a la migración, la movilidad y la «colonización» drástica en esta zona durante las últimas décadas. Eso supone una visión interesante sobre la colonización en la frontera sur de México, ya que la región ha sido conquistada y colonizada por los mismos indígenas, y de ello ha resultado también cierta (des)colonización en su sentido de modificación de las relaciones de poder entre lo indígena y lo no indígena, que alcanzó su auge con el movimiento zapatista en su levantamiento en 1994 y luego con los Acuerdos de San Andrés en 1996, con los que se buscó modificar las relaciones entre el Estado y los pueblos indígenas (véase López y Rivas 2004).

En cuestión de estudios sobre la frontera sur de México, el análisis se ha centrado más comúnmente a partir de la teoría de Frederick Jackson Turner (1893), quien inició la reflexión sobre las fronteras-frentes en el caso de la historia del oeste norteamericano como un sistema de expansión de colonos blancos hacia espacios considerados como vacíos a pesar de la población indígena que habitaba en ellos (véase Fábregas 1997, Kauffer 2002, De Vos 1993). Aquí se nota la diversa terminología en inglés para la palabra «frontera»: frontier, boundary, border, borderlands que ofrece un entendimiento amplio y colorido del mismo término. Según varios autores, a partir del estudio de Turner se ha aplicado el concepto de frontera en dos sentidos principales: 1) la frontera-frente que se expande, que pueden ser fronteras agrícolas, ganaderas o urbanas, y 2) la frontera-límite que se 
identifica con la geopolítica física: las líneas divisorias entre los países, municipios, etc., lo cual también ha sido dominante en la frontera sur de México, en su colindancia con Guatemala y Belice. Sin embargo, vale la pena señalar que la teoría de Frederick Turner Jackson ha sido cuestionada en los debates anglosajones por tener un entendimiento limitado sobre el concepto (en inglés, la diferencia entre frontier y border), como también por su trayectoria colonialista (véase Hämäläinen y Truett 2011).

Así, se puede observar que el redescubrimiento de la frontera sur de México ha propiciado una abundante cantidad de estudios enfocados en este espacio, los cuales se centraron al principio en la colonización, la migración y en la historia de la región. Los estudios nos pintan un territorio recientemente visibilizado y relativamente periférico en la vida nacional, pero también activo y movilizado en su propio ámbito.

Por otra parte, sucede que después del conflicto centroamericano y sus efectos en la frontera sur de México, el levantamiento zapatista y luego la migración internacional a partir de los noventa aumentó el interés nacional e internacional en dicha frontera, y al mismo tiempo se ha acelerado la investigación en la zona. La frontera sur ha crecido en cuestión de atención, población, actores variados e investigaciones, y de ello han resultado procesos nuevos que han marcado su historia contemporánea. Detecto como consecuencia al menos dos tendencias.

En primer lugar, la frontera sur ya no representa solo una región marginal y periférica sino una caracterizada por varios procesos políticos contemporáneos que han sido la base de no pocos estudios, nacionales e internacionales, para su entendimiento. En este sentido, se puede cuestionar el sentido periférico de la frontera sur. En segundo lugar, al parecer el estado de Chiapas se convirtió en centro de atención debido a su ubicación geopolítica (lugar de refugiados, camino de migrantes y territorio de ubicación de la selva Lacandona) y por el movimiento zapatista en su territorio. El estado, entonces, se vuelve el pilar del entendimiento de la frontera sur de México, incluso hegemónico. ${ }^{6}$

\footnotetext{
${ }^{6}$ En este artículo no he podido tratar a profundidad los otros estados de la frontera sur, lo cual parece haber sido
}

Consultando hoy los catálogos electrónicos de las bibliotecas del Ecosur y del cIESAS-Sureste, para tomar unos ejemplos, los resultados para el concepto de la frontera sur mexicana son masivos: la búsqueda con la palabra encuentra más de 1000 resultados para el catálogo del ECOSuR, y más de 300 para el del cIESAs-Sureste. La Red de Revistas Científicas de América Latina y el Caribe, España y Portugal (Redalyc) encuentra más de 1000 resultados para «frontera sur» y más de 60 para «frontera sur México». ${ }^{7}$ Una breve revisión de los títulos de los trabajos académicos demuestra, sobre todo, la popularidad del término mencionado. Evidentemente, no todos abordan ni analizan el concepto en sí, sino que lo utilizan únicamente para la delimitación de la zona explorada en los objetos del estudio, los cuales son de gran variedad. También, delimitando la búsqueda al campo de las ciencias sociales, la frontera sur aparece, sobre todo, relacionada con aquellos sobre la migración. Y esta es precisamente la que domina los estudios dedicados a las regiones fronterizas, ya que suele exponerse como un problema para los Estadosnación y las relaciones internacionales. Así ha sido también para el caso de la frontera sur de México (véase Castillo 2002).

Como ha observado Kauffer (2002, pp. 9-15), además de la tendencia a explorar la migración, tradicionalmente la perspectiva mexicana domina la frontera sur en detrimento de las perspectivas del sur. En este sentido, se podría pensar que al mismo tiempo es un término hegemónico-nacionalista que refleja la relación de dominación hacia el sur de la frontera sur. Muy posiblemente, este pensamiento recalque el propio desarrollo de la frontera sur de México y los estudios al respecto, en comparación con los anteriores sobre la región sureste en donde está comúnmente planteada como una periferia del Estado-nación mexicano debido a su posición comparativa con

una cuestión pendiente con respecto a este tema (véase, por ejemplo, Kauffer 2002). Sin embargo, para profundizar sobre tales procesos políticos contemporáneos en la frontera sur de México, y en el estado de Chiapas, sobre todo, véase por ejemplo: Leyva Solano y Ascencio Franco (1997, 2002), Villafuerte y Leyva Solano (2006), Mattiace, Hernández y Rus (2002), Leyva Solano y Burguete Cal y Mayor (2007), Pérez Ruiz (2004).

${ }^{7}$ Consultas realizadas en mayo de 2013. 
la atención de la frontera norte, que también se puede considerar como hegemónica/periférica. Son dos fronteras llenas de contradicciones pero interdependientes, lo cual se refleja también en los estudios sobre ellas. ${ }^{8}$

Debido a los procesos políticos contemporáneos, en la exploración de estudios recientes que se dedican en forma creciente a cuestiones de medio ambiente, ecología y recursos naturales se detecta una visión más transfronteriza que se articula como una necesidad para entender dichas fronteras y sus realidades vividas o construidas (García y Kauffer 2011, Villafuerte y Leyva 2006). Considero que la necesidad de la visión transfronteriza plantea la problemática del estadocentrismo de los estudios de la frontera sur de México debido, entre otras cosas, a los procesos de globalización y a la emergencia de otros actores no estatales, que pueden ser movimientos sociales, organizaciones no gubernamentales (ONG), académicos, think-tanks, activistas, migrantes indocumentados, etc. ${ }^{9}$ ¿Logra la visión transfronteriza desafiar y superar la naturaleza estadocéntrica y hegemónica de la frontera sur de México?

${ }^{8}$ En sentido geopolítico, las relaciones internacionales entre los Estados Unidos-México-América Central han vuelto a generar más interés hacia la frontera sur de México. Se puede observar cómo las dos fronteras - el Norte y el Sur - interactúan y dependen de una y otra. Si anteriormente la frontera sur era la periferia ignorada u olvidada por la importancia destacada de la frontera norte con los Estados Unidos, hoy el interés de los Estados Unidos por cuidar su frontera con México está ilustrado con la preocupación de qué pasa en la frontera sur de México, sobre todo en cuestión de los migrantes indocumentados transfronterizos, pasando por México como un territorio de frontera enorme en el camino hacia su destino final. Según Sandoval (1997: 155-163), con el proceso de la globalización y el área norteamericana de libre comercio, las fronteras nacionales cobran una nueva dimensión en términos geopolíticos y geoeconómicos en donde la frontera sur de México se ha convertido en parte de Centroamérica o una "América en medio».

${ }^{9}$ En la obra editada por Bovin (1997), se analizan las múltiples fronteras del istmo entre el sur de México y América Central. Sin embargo, el entendimiento que caracteriza el análisis suele todavía ser el de la frontera física, ya sea en su forma frente-pionero, frente-conquista, o frente-límite en relación con los Estados-nación que compiten por una frontera «ejemplar» como parte de las políticas internacionales.

\section{SOBREPASANDO LOS LIIMITES: LA TESIS DE LA GLOBALIZACIÓN}

Durante la década de los noventa tuvo lugar en las ciencias sociales un intenso debate sobre la globalización. En síntesis, se cuestionaba si como concepto indicaba una nueva era en la política. O más precisamente, si funcionaba como un concepto analítico capaz de comprender algunas prácticas, ideas, y luchas sociopolíticas que caracterizan la política internacional ¿Cambió acaso la concepción de las fronteras políticas de los Estados-nación, definidas y establecidas principalmente por las élites en la Paz de Westfalia en 1648, donde por primera vez se introdujo el concepto de soberanía nacional? ¿Es la globalización algo que deberíamos explicar o es algo que nos explica el orden mundial actual? ${ }^{10}$

En estos debates se pueden distinguir tres concepciones diferentes sobre la globalización. La primera la trata como una nueva era: una época en que se considera a los Estados-nación como un actor entre otros actores relevantes. En el trabajo clásico de Hirst y Thompson (1999) se plantea la crítica sobre el estadocentrismo de la globalización y se cuestiona el planteamiento original de Max Weber en el cual los Estados-

\footnotetext{
${ }^{10}$ Considero que el debate sobre la globalización era especialmente importante para la disciplina de las relaciones internacionales (RI), que se ubica mayormente dentro de la ciencia política. El campo de las RI se ha dedicado tradicionalmente a explorar las razones de la guerra, emergido sobre todo después de la primera guerra mundial en los Estados Unidos. Fundamentalmente, la disciplina se ha dedicado a la política exterior de los países, la diplomacia y las organizaciones internacionales. Sin embargo, como resultado del debate sobre la globalización, la disciplina de las RI recibió críticas por su estadocentrismo y eurocentrismo dados sus origines mencionados. Además de la política misma, que se considera internacional y global, se exploran también los espacios políticos que sobrepasan las fronteras de las comunidades tradicionales de las sociedades. El estadocentrismo, como también el eurocentrismo, se ha considerado erróneo por dejar fuera a otros actores importantes. Se podría decir que el debate de la globalización implicaba una cierta, aunque limitada, descolonización del estudio de las relaciones internacionales. Para profundizar sobre el desarrollo de la disciplina de las RI, la tesis de la globalización, la descolonización, el estadocentrismo y eurocentrismo, véase, por ejemplo: Gruffydd Jones (2006), Darby (2004), Hamati-Ataya (2012), Reus-Smit (2012), Devetak y Higgott (1999), Held y McGrew (2000), Saurin (1996), Kaldor (2003), Yashar (2007).
} 
nación, con su monopolio de la violencia, eran la forma dominante de gobernanza en un orden mundial que no aceptaba la competencia de otros actores. Así, la tesis de la globalización permitía argumentar que la política mundial contemporánea era policéntrica, un sistema complejo de varios poderes descentralizados. Aunque los Estados-nación se consideraban importantes, ya no eran los únicos actores del poder mundial, sino que se observaba la emergencia de otros no estatales, como las instancias internacionales de gobernanza, las empresas multinacionales, los movimientos sociales y los ciudadanos sin fronteras.

En la segunda acepción se entendía la globalización como una nueva estructura del mundo. Según este enfoque, es una estructura no estadocéntrica que no es necesariamente definida por la superioridad de unos Estados sobre otros, sino que la estructura de la política mundial estaba formada por la gobernanza o el sistema capitalista global. En el centro de este debate se encuentra el eterno interrogante sobre la estructura y la agencia (Hardt y Negri 2000). Por último, existe la concepción de la globalización como una perspectiva desde la cual se puede analizar la política mundial. Según Hirst y Thompson (1999), la tesis de la globalización como una perspectiva y herramienta de análisis tiene cierta fuerza en comparación con las visiones anteriormente explicadas, ya que desde el punto de vista global se puede analizar el rol de los Estados-nación, los cambios en el orden mundial y el significado de las fronteras sin caer en un análisis simplista sobre la desaparición de las fronteras, la decaída del papel de los Estados-nación o la omisión de los cambios en el sistema global debidos a los procesos políticos contemporáneos más allá de las fronteras estatales.

Como hemos visto, la tesis sobre la globalización se relaciona con una postura crítica y la deconstrucción de paradigmas en el marco de las relaciones internacionales, que cuestiona el estadocentrismo y replantea el análisis de la política mundial contemporánea posterior a la guerra fría y a la caída de Muro de Berlín. La base del análisis de las relaciones internacionales (RI) la conformaba un mundo bipolar marcado por fuertes líneas-fronteras entre superpoderes territoriales e ideológicos. Esta visión y la realidad de la política mundial se reflejaba en un concepto de fronteras estáticas con poca fuerza explicativa, que ya no se aplicaba en el contexto político de los noventa, marcado por la emergencia de una sociedad civil global, procesos de democratización, gobernanza internacional, integración y expansión de la Unión Europea, internet y las empresas multinacionales. El cambio del orden mundial en el examen de la política y los otros actores no estatales surgidos desde el debate de la globalización ha significado un reto especial para el campo de estudio de las RI que se refleja en la tesis de la globalización y el análisis del mundo sin fronteras que llegó a niveles algo idealizados. Por otro lado, la tesis tampoco debe analizarse de forma simplista, ya que tiene fuertes implicaciones como el cuestionamiento del estadocentrismo, el eurocentrismo y el enfoque estático sobre las fronteras.

En este sentido, retomo el análisis de Varela (1997), quien señala la ambigüedad en el concepto de frontera tradicionalmente empleado en las relaciones internacionales. Por un lado, el espacio político internacional se ha determinado por las fronteras que separan el territorio entre los Estados soberanos, y de esta forma distinguen el ámbito geográfico para el ejercicio exclusivo del poder de un Estado. En esta teoría, las fronteras son, sobre todo, líneas. Las líneas-fronteras marcan el espacio territorial geográficamente delimitado, un fenómeno internacional que establece una relación entre los Estados. Sin embargo, como argumenta Varela, esta definición preliminar ha carecido de valor explicativo: las fronteras han aparecido como un fenómeno compacto, estático y atemporal; por lo tanto, las posibilidades de análisis que introduce esta definición se agotan fácilmente. Así, agrega Varela, en el análisis de las fronteras en las RI han pasado dos cosas contradictorias. En primer lugar, han jugado un papel fundamental como principio esencial de organización internacional que delimita y distingue a los actores fundamentales, los Estados, considerados como comunidades territorialmente limitadas. En segundo lugar, su estudio ha ocupado una posición marginal pues, al considerarse como «algo dado», ha tenido poca fuerza explicativa. 
Varela destaca el valor universal de la frontera como fenómeno cuyo significado sería relativamente homogéneo a nivel mundial y que se explora solo en relación con el Estado, la soberanía y la seguridad nacional. En este sentido, se expresan fundamentalmente una relación de poder y un concepto «hegemónico». Esta concepción de frontera se ha aplicado también en otros continentes al considerarse como universal y legítima. Sobre todo durante la guerra fría, se asumía una relación directa de las fronteras con el mantenimiento del status quo y la estabilidad del orden internacional, por lo cual las disputas fronterizas - ya fueran grupos étnicos en las fronteras, la existencia de fronteras difusas etc.- eran identificadas como una factor que incrementaba el potencial de conflicto e indicadoras de inestabilidad. El estudio de las fronteras, entonces, se ha enfocado en la exploración de los conflictos que, argumenta Varela, tiene que ver con la visión primordialmente occidental de las fronteras a las cuales se atribuyen características de exclusión, exclusividad y dicotomías modernas de universalidad y racionalidad en donde no caben, por ejemplo, los grupos étnicos y los pueblos indígenas. Además, este concepto de la función de las fronteras también explica que comparativamente tenga poca importancia en el campo de las RI: adquiere relevancia principalmente en casos de conflicto o de un cambio significativo que altere su trazo original o su función. En las RI existen dos vertientes: en el primer caso, la exploración de los conflictos relacionados con las fronteras; en el segundo, el análisis de la mundialización o globalización de la economía política internacional sin fronteras, que son tendencias algo opuestas.

En esta discusión, el debate sobre la globalización sin fronteras adquiere varios significados pues: 1) cuestionó el estadocentrismo y el eurocentrismo de las RI, y su visión de la política internacional; 2) sacudió el concepto rígido de las fronteras, aunque en su fase más agitada implicó desechar las fronteras por completo; y 3) inició un análisis renovado sobre el orden mundial contemporáneo (véase también Laako sf). La perspectiva de la globalización nos permite el cuestionamiento del estadocentrismo y el nacionalismo relacionados con la frontera sur de
México, en donde, además de ser un objeto de análisis de colonización como expansión, dominación, relaciones centro-periferia y formación de la nación, nos invita a repensar el concepto en el contexto de los debates actuales, derivados de las reacciones a la tesis de la globalización.

\section{PENSAR DESDE LAS FRONTERAS: FRONTERAS A DEBATE EN LAS CIENCIAS SOCIALES}

Los geógrafos Newman (2006a, 2006b) y Paasi (2005), entre otros, encuentran que como reacción contra las tesis de la globalización ha emergido un nuevo entusiasmo científico sobre el estudio de las fronteras. ${ }^{11}$ Según Newman (2006a) se ha empezado a entender que no solamente la frontera sino su proceso de formación es lo que más afecta a las realidades vividas de las fronteras, ya sea desde los ámbitos globales y nacionales hasta niveles locales y de escala microindividual. Este mismo autor sostiene que durante la década de los ochenta emergió una nueva generación de trabajos sobre las fronteras que fueron cuestionando las tipologías de los estudios clásicos. Durante las décadas de los ochenta y los noventa el análisis se enfocó en la cooperación y las regiones transfronterizas, con un fuerte peso en Europa y la apertura de fronteras con motivo de la Unión Europea. A diferencia de su anterior sentido geográfico, ya no se concebía solo como una barrera sino como un espacio de reconciliación, cooperación y coexistencia. Durante la misma época irrumpió el debate sobre la globalización contra las fronteras-barreras, según Newman, apoyado sobre todo por los economistas e informáticos, para quienes el mundo sin fronteras se encontraba en la Europa Occidental. Sin embargo, como reacción a ello, los geógrafos, politólogos, sociólogos y abogados empezaron a cruzar sus propias fronteras disciplinarias para repensar

\footnotetext{
${ }^{11}$ Para profundizar sobre los estudios de la frontera o las regiones fronterizas (Border Studies / Borderlands Studies), sobre la relevancia contemporánea del concepto de la frontera, las reacciones a la tesis de la globalización así como los debates sobre género y otredad etc., recomiendo revisar varios números y artículos de las revistas Geopolitics y Journal of Borderlands Studies. Véase, por ejemplo, el número especial de Geopolitics (2005, v. 10), Banerjee y Ray (2012), Singleton (2008), Huesca (2004).
} 
los significados de las fronteras, no solamente las de Europa Occidental, sino las que dividen al Primer Mundo y al Tercer Mundo (sobre todo, la frontera entre los Estados Unidos y México), las fronteras de imposición y de reconfiguración en África, y la frontera entre Israel y Palestina. Según Newman (2006a), se volvía a la pregunta básica: «¿Por qué algunos procesos -incluso los de globalización- pasan en algunos lugares pero no en otros? ¿Por qué están desapareciendo algunas fronteras al mismo tiempo que otras están en proceso de construcción como manifestaciones físicas de un paisaje político?». ${ }^{12}$

Newman $(2006 a, 2006 b)$ identifica dos formas principales de entender las fronteras en los estudios contemporáneos. En primer lugar, el entendimiento de la frontera como un proceso o una institución. Básicamente, se trata del reconocimiento de que la frontera no es resultado solamente de algo sino un proceso de formación de algo, muchas veces una decisión tomada por las élites para determinar hasta qué punto una frontera debería abrirse o cerrarse. La pregunta sobre su apertura o cierre es una tendencia significativa en los estudios sobre las fronteras: por un lado, se ha reconocido que algunas fronteras se han vuelto flexibles y permeables pero, por otro lado, después del 11 de septiembre de 2001 se ha encontrado un nuevo paradigma que reenfoca la atención en el proceso de control y cierre de las fronteras, más claramente observado en las fronteras de los Estados UnidosMéxico-Canadá. Según Newman, una gran parte del financiamiento de la investigación sobre las fronteras después de los ataques terroristas se ha destinado a la búsqueda de formas más efectivas de control. El segundo aspecto que identifica Newman es la frontera como un espacio de transición que explora las interacciones en las zonas fronterizas o transfronterizas. Este estudio nace

${ }^{12}$ La crítica de Newman (2006b) hacia la tesis de la globalización sin fronteras es que la teoría de transición de un mundo con fronteras a uno sin fronteras hubiera indicado una transferencia automática de poder desde un grupo de interés a otro. En este sentido, el acto de hacer desaparecer fronteras no es menos (neo)colonialista que la imposición anterior de las fronteras-barreras. En este sentido también nota que aunque la tesis de la globalización percibe las fronteras como redes, contactos, movimientos y flujos, y la existencia de «una sociedad mundial», no todos los ciudadanos del mundo son miembros de ellos. a contracorriente del entendimiento de las fronteras como barreras. Así, se perciben las fronteras como puentes de interacción, como la mixtura de culturas y la hibridad de identidades bajo el argumento de que no todas las fronteras existen según las líneas territoriales entre los Estadosnación sino, por ejemplo, dentro de las grandes metrópolis como Londres o Nueva York. ${ }^{13}$

El antropólogo Álvarez (1995) nota en su famoso estudio que las regiones fronterizas y las fronteras se volvieron populares en las ciencias sociales y las humanidades después de la segunda guerra mundial. Él define su Borderland como una región y un conjunto de prácticas definidas por la frontera, la cual está caracterizada por conflictos y contradicciones, ya sean materiales o ideales. Argumenta que la frontera entre México y los Estado Unidos forma la base principal para la emergencia de los estudios sobre las regiones fronterizas. Anteriormente, estas eran percibidas como lugares desérticos y como una línea entre dos culturas grandes (en este caso, la mexicana y la anglosajona). Los estudios sobre la frontera también tenían la tendencia a visualizar estas regiones como «congeladas en el tiempo", con población analizada desde el esencialismo. Pero el entendimiento sobre las fronteras se expandió junto con la emergencia de las ciudades fronterizas que se convirtieron en «laboratorios»: mezclas de poblaciones e identidades en muchos sentidos. Por último, surgió la visión de la región fronteriza relacionada con las políticas públicas en cuestiones de migración,

13 Aquí también se presenta la definición básica de Borderlands, las regiones fronterizas, que no se refiere solamente a la línea divisoria sino al área cerca de la frontera con sus propias dinámicas y prácticas de la vida cotidiana afectadas por la proximidad de la frontera. Para el geógrafo Paasi (2005) la exploración de las fronteras ha sido definida por el análisis del contexto. En este sentido, las fronteras son siempre únicas y particulares, caracterizas por sus contextos de lugares concretos y contextuales. Es el contexto que es significante en el caso de las fronteras: las fronteras son únicas, pero siempre relacionadas con sus diferentes formas a procesos locales, regionales, nacionales y supranacionales. El contexto y su relación con los procesos que les marcan a las fronteras, tienen siempre un aspecto politico en ellos: las fronteras tratan del poder, de discursos, de prácticas, emociones, memorias y expectativas del futuro, lo cual les brinda un sentido y grado de politización. 
trabajo, nuevas poblaciones y sus impactos. ${ }^{14}$ Según Álvarez, la última gran transformación en los estudios de las fronteras en antropología es la emergencia de las «voces nativas»: las voces desde el interior de las regiones fronterizas más allá de la interpretación del «otro». En primer lugar, afloró la tendencia del «folklore», como la cultura popular mexicana-texana con sus $\mathrm{CO}^{-}$ rridos. En segundo lugar, irrumpieron las voces de las mujeres chicanas que le dieron un nuevo sentido a las fronteras al cruzar conflictivamente la frontera como un factor desestabilizador del status quo, la frontera como un contexto de conflicto y de opresión. Esta es una tendencia en los estudios de la frontera, en donde los propios sujetos fronterizos hablan de sus experiencias y realidades..$^{15}$

El concepto de frontera de la escritora-investigadora feminista-chicana Anzaldúa (1987) es muy complejo e ilustrativo, y lo retomo aquí como un ejemplo de las tendencias giradas hacia las voces nativas de las regiones fronterizas y de género. Su uso de la frontera se postula desde su identidad como mujer de color, con herencia mestiza-india-texana, como lesbiana sujeta a triple-discriminación, y que vive concretamente en la región de la famosa frontera entre México y los Estados Unidos, consciente de su posición fronteriza y de su lenguaje fronterizo-chicano:

\footnotetext{
${ }^{14}$ Por ejemplo, los antropólogos han explorado las implicaciones de las maquiladoras en la frontera norte de México. Es un factor que tal vez explique la última tendencia percibida para la visión transfronteriza en la frontera sur de México. Según Álvarez, lo importante de la investigación de la frontera ha sido el hecho de documentar la vida en las fronteras. De ahí, analiza Álvarez, la migración se convirtió en un eje significativo de análisis, lo cual desafió la interpretación parroquial de las comunidades territoriales. En el centro de análisis se pusieron las vidas de los migrantes y sus relaciones extendidas hacia otros lugares. $\mathrm{Al}$ mismo tiempo, creció el enfoque de economía globalizada que influyó en el crecimiento de los estudios en la migración. Si me aventuro un poco, la antropología también pareciera estar algo influida por la tesis de la globalización.

${ }^{15}$ Según Álvarez (1995), el estudio de fronteras ha experimentado un giro de análisis hacia extensiones metafóricas que capturan la imaginación. Se podría decir incluso que hoy en día el concepto de la frontera y la región fronteriza capta igualmente la imaginación que la tesis de la globalización lo hacía antes: ambos permiten un toque místico y amplio de varios sentidos. Existe la posibilidad de que el estudio de las fronteras a veces caiga en ser elusivo, indefinido, tautológico y mitificado.
}

entre lo hegemónico y lo periférico. ${ }^{16}$ Según Saldívar (2007), la frontera transnacional de Anzaldúa ha representado un giro importante en el entendimiento de la frontera que es marcadamente diferente al autor clásico de las fronteras, el anteriormente mencionado Frederick Jackson Turner, aunque ambos abordan condiciones subalternas: para Anzaldúa, la frontera es la colonización y la migración de reversa y no lo universal; es el principio más bien que el final. Y es la conciencia fronteriza la que, según Mignolo (2000), en vez de intentar dividir el mundo en forma dicotómica occidental, trata de ir a estas fronteras dicotómicas para analizar las fronteras desde ahí. Saldívar argumenta (2007) que Anzaldúa invoca una nueva conciencia,

${ }^{16}$ La obra de Anzaldúa se puede considerar como ejemplo de las tres últimas tendencias identificadas sobre los estudios de las fronteras y, sobre todo, del estudio de las regiones fronterizas: la de las voces nativas, la de narrativas fronterizas, la de género y la de literatura. Aunque la obra de Anzaldúa ha agitado una cantidad respetable de reacciones y análisis sobre las fronteras, es también observable que estas mismas fronteras siguen siendo las menos exploradas (Segura y Savella 2008, Saldívar 2007, Keating 2006). Analizando brevemente la contribución de la obra de Anzaldúa para el entendimiento de la frontera, se pueden identificar por lo menos los siguientes significados. En primer lugar, la frontera aparece como un lugar o una región concreta en medio de algo: entre México y los Estados Unidos con sus respectivas culturas. Al mismo tiempo, la frontera —el lugar, la región- se ubica en los márgenes de estas dos culturas y países. En este sentido, la frontera es el punto de encuentro, la encrucijada y la coyuntura de estas culturas y países. Pero también - esto es la contradicción y el conflicto- es el lugar y la región de la periferia de las dos culturas y países: el espacio de sombra, el territorio desconocido, extraño y no purista. Es la mezcla, lo mixto, lo no auténtico de ambos lados. Argumenta Anzaldúa que la región fronteriza siempre plantea la pregunta sobre la autenticidad por su carácter de mezcla. En este sentido, para sus habitantes — reales o imaginarios - la frontera representa una herida abierta. Es una herida abierta por su naturaleza dual que no es auténtica en ningún lado aunque consta de elementos de ambos lados. De ahí, la formación de su propia cultura e identidad aunque sea contradictoria y poco entendida. Sin embargo, la frontera es siempre un camino hacia algo, no estático y no esencialista que requiera una conciencia. La conciencia fronteriza, para Anzaldúa, aparece como un conocimiento particular que requiere una salida -intelectual o concreta- a un lugar desconocido, de posicionarse de forma distinta, conquistar el espacio no confortable a uno mismo y transformar la forma de pensar sobre la región fronteriza. Así, la conciencia fronteriza también es una inquietud, una inquietud hacia algo nuevo que no puede ser mantenido dentro de fronteras rígidas de los conceptos. 
pensamiento y emergencia de lo sureño y norteño, entre los llamados Primer y Tercer Mundo.

Interpreto que en estos debates recientes subyace un cuestionamiento profundo que remarca el entendimiento anterior de la frontera enfocada en la idea de colonización, y que vislumbraba las diferencias entre supuestos centros y periferias geográfico-nacionales. El cuestionamiento emergiendo desde los debates de los Borderlands Studies o Border Studies pudiera permitirnos también repensar los significados de la frontera sur de México: ¿Qué tan periférica sigue siendo esta frontera?

\section{REFLEXIONES FINALES: LA FRONTERA SUR DE MÉXICO DESDE LA PERSPECTIVA DE LOS ESTUDIOS DE REGIONES FRONTERIZAS}

En este artículo he explicado cómo la tesis de una globalización sin fronteras ha servido para generar un interés renovado en los estudios sobre las fronteras y las regiones fronterizas. Según la literatura contemporánea, el debate tiene que ver con la exploración de las «orillas de imperios, naciones y sistemas-mundo» (Hämäläinen y Truett 2011). Para los autores anteriormente mencionados, la perspectiva de los estudios de frontera y de las regiones fronterizas puede concebirse como forma de «trascender los puntos ciegos (del enfoque) centrista» $y$ «privilegiar micro-historias», de tal forma que se constituya en un campo de estudio de contranarrativas que desafíen las percepciones dominantes (Hämäläinen y Truett 2011: 338-339).

Aun así, Hämäläinen y Truett (2011: 357) advierten el riesgo de que los estudios de frontera refuercen viejas dicotomías: «Tenemos que evitar caer en la trampa de visualizar las tierras fronterizas en contraposición a los territorios centrales de naciones e imperios. De hecho, los puntos cruciales de las narrativas centrista, no son siempre lo que la gente experimenta en los lugares céntricos o tampoco son siempre lo que la gente experimenta en los lugares centrales» (traducción mía). Del mismo modo, Lakomäki (2014: 3) señala que:

incluso entre los estudiosos de las fronteras ha predominado la tendencia a enfocarse en fronteras y naciones euroamericanas. En cambio, los pueblos indígenas se han retratado a menudo como 'pueblos en medio de bordes fronterizos', habitando regiones fronterizas porosas entre imperios y estados rivales, en lugar de ser considerados como naciones que habitan sus territorios enmarcados por fronteras propias. Mientras los estudios sobre fronteras han, en gran medida, permitido avanzar nuestro entendimiento sobre las estrategias geopolíticas de pueblos nativos durante la era del colonialismo europeo, en ocasiones corren el riesgo de negar el modo complejo en que los pueblos indígenas se conciben a sí mismos como naciones en el centro de su mundo (traducción mía).

Retomando estos debates actuales de los estudiosos de las regiones fronterizas, argumento que sería fructífero para las discusiones teóricas sobre la frontera sur de México vincularse con estas cuestiones de repensar el vocabulario y el imaginario sobre la región fronteriza mencionada, descrita anteriormente con términos como redescubrimiento, periferia, colonización, expansión, un lugar exótico/distante etc. (Laako sf). ¿Pueden las regiones fronterizas ser analizadas más allá del centro y la periferia? ¿Pueden estas visiones estar impugnadas dentro de la frontera sur de México? ¿Hasta qué punto reproducimos las visiones colonialistas desde la perspectiva de las regiones fronterizas? Para futuros debates sobre el significado de esta frontera sería interesante plantear de manera paralela estos debates.

En este artículo he tenido el objetivo de repasar los debates sobre la frontera sur de México desde el enfoque de realidad vivida como línea física entre países de la región sureste, y como una construcción y un concepto debatido por parte de las instancias académicas y de los investigadores a partir de la década de los ochenta. En este análisis se observa una frontera sur de México retomada como una región relativamente periférica y olvidada en comparación con su contraparte dominante del norte, pero no sin sus procesos de colonización, migración y movilización indígena, caracterizada, sobre todo, con la entrada de los refugiados guatemaltecos.

Por el otro lado, las transformaciones políticas de las últimas décadas han modificado tanto la propia frontera sur como los estudios sobre ella, marcada ahora por los sucesos tanto en la fron- 
tera norte como en Centroamérica. En este contexto, emergen también invocaciones por una visión transfronteriza, que aunque siga caracterizada por la necesidad primordial de resolver conflictos o problemáticas fronterizas - reales o potenciales-, extiende al mismo tiempo nuestro entendimiento anterior como frontera-barrera. Observo que el desarrollo de los debates sobre el concepto de la frontera ha ilustrado los contextos políticos en los que nacen también los mismos entendimientos sobre la frontera.

Como el debate sobre las fronteras lo demuestra, la politización de la tesis de globalización sin fronteras dentro del mismo debate académico nos hizo tomar distancia crítica del entendimiento estático de la frontera como barrera física, lo que suele producir investigaciones descriptivas con poca fuerza explicativa y, al mismo tiempo, nos ha permitido cuestionar y repensar la visión nacionalista, estadocéntrica y eurocéntrica de este entendimiento de la frontera. Aunque estas visiones siguen siendo útiles, los cambios políticos y el contexto en que analizamos las fronteras nos afecta y nos obliga a ampliar el entendimiento de la frontera que estamos explorando para destacar mejor las realidades vividas y construidas. Asimismo, los debates críticos recientes sobre las regiones fronterizas dentro de las ciencias sociales - ampliamente abordados en este artículo- nos pueden hacer repensar la visión construida sobre la frontera sur de México.

\section{FUENTES DE CONSULTA}

Álvarez Junior, Robert R., 1995, «The Mexican-US Border: The Making of an Anthropology of Borderlands», Annual Review of Anthropology, 24, pp. 447-70.

Anzaldúa, Gloria, 1987, Borderlands / La Frontera: The New Mestiza, San Francisco: Aunt Lute Books.

Banerjee, Paula y A. B. Ray Choudhury, 2012, «Introduction: Women in Indian Borderlands», Journal of Borderlands Studies, 27(1), pp. 27-29.

Bovin, Philippe, 1997, Las fronteras del Istmo: fronteras y sociedades entre el Sur de México y América Central, México: CiEsAs.

Castillo, Manuel Ángel, 2002, «Región y frontera: la frontera sur de México», en Edith Kauffer (ed.), Identidades, migraciones y género en la frontera sur de México, México, Ecosur, pp. 19-49.
Chenaut, Victoria, 1989, Migrantes y aventureros en la frontera sur, México, SEP/CIESAS.

Darby, Phillip, 2004, «Pursuing the political: a postcolonial rethinking of relations international», Millennium: Journal of International Studies, 33(1), pp. 1-32.

Devetak, Richard y Richard Higgott. 1999. «Justice unbound? Globalization, states and the transformation of the social bond», International Affairs, 75(3), pp. 483-498.

Fábregas Puig, Andrés, 1997 a, Ensayos Antropológicos 1990-1997, México, Gobierno de Chiapas y UNICACH.

_ 1997b. «Prólogo», en Xóchitl Leyva y Gabriel Ascencio, Colonización, cultura y sociedad, México, UNICACH.

——, 2011, Configuraciones regionales mexicanas: un planteamiento antropológico, México, UNICH.

Fábregas Puig, Andrés, Juan Pohlenz, Mariano Báez y Gabriel Macías, 1985, «La formación histórica de la Frontera Sur», México, Ciesas-Sureste (Cuadernos de la Casa Chata, 124).

García, Antonino y Edith Kauffer, 2011 «Las cuencas compartidas entre México, Guatemala y Belice: Un acercamiento a su delimitación y problemática general», Frontera Norte, 23(45), pp. 131-161.

Geopolitics, 2005, «Contemporary Border Realities», v. 10 , pp. 680-687.

Gruffydd Jones, Branwen, 2006, Decolonizing International Relations, Lanham: Rowman y Littlefield Publishing.

Hämäläinen, Pekka y Samuel Truett, 2011, «On Borderlands», Journal of American History, 98(2), pp. 338-361.

Hamati-Ataya, Inanna, 2012, «IR Theory as International Practice/Agency: A Clinical-Cynical Bourdieusian Perspective», Millennium: Journal of International Studies 40(3), pp. 625-646.

Hardt, Michael y Atonio Negri, 2000, Empire, Cambridge, Harvard University Press.

Held, David y Anthony McGrew, 2000, The Global Transformations Reader, Cambridge, Polity Press.

Hirst, Paul y Grahame Thompson, 1999, Globalization in Question, Cambridge, Polity Press.

Huesca, Robert, 2004, "Cross-border justice movements and maquiladora workers», Journal of Borderlands Studies, 19(2), pp. 45-76.

Jackson Turner, Frederick, 1893, «The Significance of the Frontier in American History", www. milestonedocuments.com/documents/ view / frederick-jackson-turner-the-significance-of-the-frontier-in-american-histo/text [consultado: noviembre 6, 2014]

Kaldor, Mary, 2003, «The Idea of Global Civil Society», International Affairs, 79(3), pp. 583-593. 
Kauffer, Edith, 2002, Identidades, migraciones y género en la frontera sur de México, México, Ecosur.

Keating, Ana Louise, 2006, «From Borderlands and New Mestizas to Nepantlas and Nepantleras: Anzaldúan theories for social change», Human Architecture: Journal of the Sociology of SelfKnowledge, 4(3), pp. 5-16.

Keck, Margaret y Kathryn Sikkink, 1998, Activists beyond borders: advocacy networks in international politics, Ithaca, Cornell University Press.

Laako, Hanna, s.f., «From Borderless Globalization towards Decolonizing Vision: The Mexican Southern Borderlands in Critical Review» [en dictamen].

Lakomäki, Sami, 2014, Gathering Together: The Shawnee People through Diaspora and Nationhood, 16001870, New Haven-Londres, Yale University Press.

Leyva, Xóchitl y Gabriel Ascencio, 1997, Colonización, Cultura y Sociedad, México, Unicach.

, 2002, Lacandonia al filo del agua, 2a ed., México, CIESAS.

Leyva, Xóchitl y Araceli Burguete Cal y Mayor, 2007, La remunicipalización de Chiapas: lo político y la politica en tiempos de contrainsurgencia, México, CIESAS.

López y Rivas, Gilberto, 2004, Autonomía: Democracia o contrainsurgencia, México, Era.

Mattiace, Shannon, Aída Hernández y Jan Rus, 2002, Tierra, libertad y autonomia: impactos regionales del zapatismo en Chiapas, México, CIESAs.

Mignolo, Walter, 2000, Local Histories/Global Designs: Coloniality, Subaltern Knowledges and Border Thinking, New Jersey, Princeton University Press.

Newman, David, 2006a, «Borders and bordering: Towards an interdisciplinary dialogue», European Journal of Social Theory, 9(2), pp. 171-186.

_ $2006 b$, «The lines that continue to separate us: borders in our 'borderless' world», Progress in Human Geography, 30(2), pp. 143-161.

Paasi, Anssi, 2005, "Generations and the 'Development' of Border Studies», Geopolitics, 10(4), pp. 663-671.

Pérez Ruiz, Maya Lorena, 2004, Tejiendo historias: tierra, género y poder en Chiapas, México, INAH.

Pohlenz, Juan, 1985, «La conformación de la Frontera entre México y Guatemala. El caso de Nuevo Huixtán en la Selva Chiapaneca», en Andrés Fábregas et al., La formación histórica de la Frontera Sur, México, cIEsAs-Sureste, pp. 25-131.

Reus-Smit, Christian, 2012, «International Relations -Irrelevant? Don't blame theory». Millennium: Journal of International Studies, 40(3), pp. 525-540.
Saldívar, Antonio y Arturo Arreola, 1997, «Colonización y desarrollo en la Frontera Sur. Perspectivas de un modelo en crisis», en Xóchitl Leyva y Gabriel Ascencio, Colonización, Cultura y Sociedad, México, UnicACH, pp. 235-244.

Saldívar, José David, 2007, «Unsettling race, coloniality and caste», Cultural Studies, 21(2-3), pp. 339-367.

Sandoval, Juan Manuel, 1997, «La región fronteriza del Sur de México en la perspectiva de la seguridad nacional estadounidense», en Phillippe Bovin (ed.), Las fronteras del Istmo: fronteras $y$ sociedades entre el Sur de México y América Central, México, ciesas, pp. 155-163.

Saurin, Julian, 1996, «Globalization, poverty and the promises of modernity», Millennium: Journal of International Studies, 25(23), pp. 657-680.

Segura, Denise y Patricia Zavella, 2008, «Introduction: Gendered Borderlands», Gender and Society, 22(5), pp. 537-544.

Singleton, Sara, 2008, « Not our borders': Indigenous people and the struggle to maintain shared cultures and politics in the post-9/11 United States», Journal of Borderlands Studies, 23(3), pp. 39-54.

Sohi, Seema, 2011, «Race, Surveillance, and Indian Anticolonialism in the Transnational Western U.S.-Canadian Borderlands», The Journal of American History, 98(2), pp. 420-436.

Varela, Hilda, 1997, «Definición de convergencias y divergencias a partir de las fronteras. Estudio comparado: África occidental-Istmo», en Phillippe Bovin, Las fronteras del Istmo: fronteras y sociedades entre el Sur de México y América Central, México, ciesas, pp. 55-65.

Villafuerte, Daniel y Xóchitl Leyva, 2006, Geoeconomía y geopolitica en el área del Plan Puebla-Panamá, México, ciesas / Porrúa.

Vos, Jan de, 1993, Las fronteras de la frontera sur: reseña de los proyectos de expansión que figuraron la frontera entre México y Centroamérica, México, CIESAs.

—_, 2002, «La frontera sur y sus fronteras: una visión histórica», en Edith Kauffer (ed.), Identidades, migraciones y género en la frontera sur de México, México, Ecosur, pp. 49-69.

Yashar, Deborah, 2007, "Resistance and Identity Politics in an Age of Globalization», The Annals of the American Academy of Political and Social Science, 610(160), pp. 160-181.

Fecha de recepción: 7 de febrero de 2014 Fecha de aceptación: 15 de septiembre de 2014 